disease, and to B lymphocyte reactions in this disease.

\section{Esterase and Acid Phosphatase in the Identification of Cell Type in Leukaemia}

V. JAMES AND P. W. PRICE (University of Sheffield) Since the evolution of different treatment regimes for the various leukaemias, it has become more important to make the initial classification as accurate as possible. The identification of cell type in leukaemia based only on the appearances of Romanowsky-stained blood and bone marrow films aided by the well established cytochemical reactions (peroxidase, PAS etcetera), can be difficult and often unreliable.

Esterase histochemistry can provide valuable assistance towards the identification of granulocytes, monocytes, lymphocytes, and their precursors ${ }^{1}$. Using a variety of naphthol esters as substrate various patterns were obtained. The best differentiation between granulocytes and cells of the monccyte series was observed following the sequential use of naphthol AS-D chloroacetate and $\alpha$-naphthol acetate, employing azocouplers of different colours. Applying these techniques, we were able, in some cases, to make diagnoses which could not have been reached without the results of esterase histochemistry.

Seven isoenzymes of acid phosphatase have been isolated from human leucocytes, of which isoenzyme 5 alone exhibits marked resistance to inhibition by $0.05 \mathrm{M}$ $\mathrm{L}(+)$ tartaric acid. Isoenzyme 5 is demonstrable in the abnormal cells in 'hairy cell' leukaemia whereas most other haemopoietic cells do not contain cytochemically detectable amounts ${ }^{2}$. We have demonstrated tartrate-resistant acid phosphatase in the abnormal cells of a number of patients previously diagnosed as suffering from one of the lymphoproliferative malignancies, thereby establishing the diagnosis of 'hairy cell' leukaemia in these cases.

In the light of these findings we feel that increased use of these techniques would enable accurate diagnoses to be reached in more patients.

\section{References}

${ }^{1}$ Li, C. Y., Lam, K. W., and Yam, L. T. (1973) Esterases in human leukocytes. J. Histochem. Cytochem., 21, 1-12.

${ }^{2}$ Yam, L. T., Li, C. Y., and Finkel, H. E. (1972) Leukaemic reticuloendotheliosis: the role of tartrate-resistant acid phosphatase in diagnosis and splenectomy in treatment. Arch. intern. Med., 130, 248-256.

\section{The Heparin-thrombin Clotting Time in Clinical Medicine}

J. R. O'BRIEN, M. ETHERINGTON, AND P. LAWFORD (Central Laboratory, Portsmouth) We report that the heparinthrombin clotting time of plasma is abnormally long in all cases of idiopathic thrombocytopenic purpura studied and abnormally short in diffuse intravascular coagulation. This suggests that this test, the heparin-thrombin clotting time, is influenced by platelet turnover and supports the concept that platelets are disposed of in an entirely different manner in these two conditions. These findings also suggest that a short heparinthrombin clotting time may reflect intravascular platelet breakdown and depend on liberated platelet factor 4 .

The heparin-thrombin clotting time is shorter than normal in patients after recovery from myocardial infarction, in atherosclerosis with no history of myocardial infarction, in patients with a history of deep vein thrombosis, and in an assorted group of patients with malignant disease. In the first patient with recurrent pulmonary embolization studied in detail heparin and probably aspirin normalized the heparin-thrombin clotting time. It seems this test may have an important place in assessing platelet abnormalities in vascular disease.

\section{The Kidney in Diabetes Mellitus}

D. B. BREWER (Department of Pathology, University of Birmingham) The renal lesions characteristically associated with diabetes mellitus are: (1) ArmanniEbstein change; (2) necrotizing papillitis; (3) diabetic glomerulosclerosis.

Armanni-Ebstein change, deposition of glycogen in the tubules, although very specific, appears to be of no functional significance.

Necrotizing papillitis is an important and serious complication. It appears to result from a combination of urinary tract infection and diabetes. It accounts for a large proportion of all cases of necrotizing papillitis seen at necropsy. The figures vary from 44 to $73 \%$ but will obviously vary with the incidence of other causes of necrotizing papillitis, such as analgesic nephropathy.

The glomerular changes are very common. The important lesions are the nodular and diffuse forms of diabetic glomerulosclerosis. Although they are morphologically distinct they probably result from the same underlying process음 The incidence of the changes increases: with the duration of the diabetes.

The clinical manifestations of these glomerular changes are proteinuria $\frac{\bar{\sigma}}{\sigma}$ oedema, hypertension, and renal failure $\bar{s}$ However, the correlation between the histological appearances and clinicap manifestations is not very close although in general, patients with the most severeglomerular damage have the most severe clinical manifestation.

Similarly, the correlation both betweer prognosis and rate of progression of the renal disease and the extent of the glomerular damage as seen in the initiat biopsy is not very good. Although as would be expected, in general, those witho the most severe lesions have the wors prognosis.

The Freeze-etching Approach to the Study of Islet Pathophysiology in Diabetes

L. ORCI (Institute of Histology and Embry $\frac{\mathbb{D}}{\overline{8}}$ ology, University of Geneva, Switzerland市 Freeze-etching was used to investigate possible abnormalities of membrgne systems in islet cells from diabetic Chitise hamsters. Age- and sex-matched anirals were classified according to their pas history as 'control' (aglycosuric), non ketotic diabetic, and ketotic diabeti respectively. Comparison of islet cells bo thin-section and freeze-etch electron microe scopy revealed that A-cells could b $\overrightarrow{\delta^{+}}$ unambiguously identified in freeze-etching by the presence of characteristic bundles of thick filaments in the perinuclear region In fracture faces of the plasma membran of B-cells, obvious alterations were found in the number, size, and distribution of membrane-associated particles. In B-cel]. from 'control' animals, these particles $\left(1217 \pm 29 / \mu^{2}\right)$ were distributed in क random pattern and had a mean size of $95.0 \pm 0.6 \AA$. In B-cells from diabetio and ketotic animals, the particles wer aggregated leaving completely smoot areas in the membrane; their mean number and size were also altered (767 $60 / \mu^{2} ; 102 \cdot 1 \pm 1 \cdot 1 \AA$ in ketotic animals $\mathrm{p}<0.005$ for both values). In addition to these changes in the plasma membranes an augmentation in the number of nucles pores was observed in A- and B-cells diabetic and ketotic animals. These findings point to alterations in membrane systems as possible determinants for insular dysfunction in diabetes mellitus. 